\title{
DISCUSSÃO SOBRE A AVALIAÇÃO ABERTA, NO ÂMBITO DA CIÊNCIA ABERTA
}

Discussion about open peer review in open science

\author{
Milton Shintaku \\ Ronnie Fagundes de Brito \\ Rui Seabra Ferreira Junior \\ Benedito Barraviera
}

\section{COMO CITAR}

SHINTAKU, Milton et al. Discussão sobre a avaliação aberta, no âmbito da Ciência Aberta. In: ABEC MEETING, 2019, Fortaleza. Anais... São Paulo: Associação Brasileira de Editores Científicos, 2019. http://dx.doi.org/10.21452/abecmeeting. 2019.214

\section{RESUMO}

A publicação das descobertas científicas muda periodicamente em decorrência da presença maciça e impactante da Tecnologia da Informação e Comunicação (TIC), bem como seus processos, incluindo os movimentos de "abertura". A Ciência Aberta tem se mostrado um caminho inovador e interessante por englobar diversas iniciativas, entre elas a "Avaliação Aberta pelos Pares" (open peer review). Esta última promove uma discussão acalorada entre os pesquisadores e Editores, em decorrência principalmente dos conflitos de interesse existentes. 0 presente estudo teve por objetivo avaliar a literatura escassa e disponível sobre a Avaliação Aberta pelos Pares, de forma a identificar conceitos e tendências visando apoiar os Editores na implantação desta nova modalidade de avaliação. Este assunto é pertinente e desafiador num momento em que o mundo da "velocidade" se apresenta como um novo paradigma para todos envolvidos na editoração científica. Ainda que teoricamente apresente vantagens, na medida que muda drasticamente procedimentos tradicionalmente aceitos, entre eles a avaliação duplo-cega, a abertura dos conteúdos e resultados obtidos é uma realidade e tem impactado drasticamente a produção do conhecimento científico. É possível que esta "abertura" seja irreversível haja vista que cerca de $70 \%$ da ciência publicada no mundo não tem reprodutibilidade. A abertura dos dados científicos e dos avaliadores daria transparência e seria uma maneira de amenizar o enorme dispêndio de investimentos públicos na pesquisa científica sem retorno à comunidade.

\section{PALAVRAS-CHAVE}

Ciência Aberta. Fluxo editorial. Avaliação pelos pares. Avaliação aberta. Periódicos científicos. 


\section{ABSTRACT}

The publication of scientific discoveries changes periodically as a result of the massive and impactful presence of Information and Communication Technology (ICT), as well as its processes, including "opening" movements. Open Science has shown an innovative and interesting path for several initiatives, including the Open Peer Review. The latter promotes a heated discussion between researchers and editors, resulting mainly from existing conflicts of interest. The present study aimed to evaluate the scarce and available literature on Open Peer Evaluation, in order to identify concepts and trends for using Editors in the implementation of this new evaluation modality. This subject is quite pertinent and challenging at a time when the world of "speed" presents a new paradigm for everyone involved in scientific editing. Although theoretically has advantages, as drastically changes traditionally accepted procedures, including a double-blind assessment, an openness of the contents and results obtained is a reality and has a drastic impact on the production of scientific knowledge. It is possible that this "opening" is irreversible, since about $70 \%$ of the published science in the world has no reproducibility. Openness of scientific data and evaluator would provide transparency and would be a way to soften the huge public investment in scientific research without returning to the community.

\section{KEYWORDS}

Open Science. Editorial flow. Perr review. Open review. Journals.

\section{INTRODUÇÃO}

A abertura das ciências, iniciada com o projeto ainda vigente ArXiv em 1991, tem alterado a comunicação científica em vários aspectos. Assim, tem-se os Arquivos Abertos (Open Archives), acesso aberto (Open Access), Dados Abertos (Open Data) e Ciência Aberta (Open Science), como destaques nos movimentos de abertura das ciências. Esses movimentos tiveram uma maior divulgação com o movimento de acesso aberto (Open Access), para a liberação gratuita das publicações científicas, de forma a levar a algumas revistas a mudarem o modelo de negócio na publicação, tanto que no Brasil tem se tornado um padrão publicar em Acesso Aberto.

Entretanto, pode-se afirmar que o movimento mais impactante nos processos científicos é a Ciência Aberta, por não se restringir a um único aspecto da comunicação científica, como no caso dos outros movimentos. Como relata Albagli, Clínio e Raychtock (2014), a Ciência Aberta é um guarda chuva que agrega práticas, significados e iniciativas voltados a tornar a ciência mais democráticas e participativa, com a valorização de todos que estão envolvidos em fazer ciência, mesmos àqueles que são considerados como leigos.

A ideia de uma ciência mais aberta e democrática não é tão recente, tanto que Cole (1993) apresenta o cenário do final do século passado nas pesquisas americanas, defendendo mudanças em suas práticas, visto as mudanças sócio-políticas, na necessidade de fazer mais com menos. David (1994) previra que a então ciência aberta seria uma revolução nas ciências, ante as mudanças ocorridas. Da mesma forma em que Partha e David (1994), advogam que as condutas da ciência aberta são mais adequadas ao momento em que ocorre restrições de recursos, estando em que os cientistas estão mais preparados para operar em situações mais restritivas.

Mais recentemente, Milham e Klein (2019) advogam que a Ciência Aberta (CA) fomenta a prática 
de compartilhamento de recurso, que pode diminuir a discrepância entre os que possuem maior financiamento e quem recebe menos, visto que pesquisa de ponta é um item expensicioso. Nesse mesmo caminho Banks et al (2019) salienta a necessidade de promover o compartilhamento na CA para melhoria da qualidade de resultados de pesquisa, apelando aos editores que se engajam em práticas da CA, como o pré-registro e o compartilhamento de dados, assim como estudos para verificação da eficácia das avaliações pelos pares.

Entretanto, um dos aspectos da CA que ainda provocam discussões é a Revisão Aberta pelo Pares (RAP) ou em inglês Open Peer Review (OPR), como explica Silva (2019), pelo medo de represálias e possíveis críticas, que podem afetar a vida acadêmica, mesmo que traga aspectos positivos de transparência. Esse ponto pode afetar a adesão dos editores a uso desta modalidade de avaliação, mantendo a avaliação tradicional de dupla cega, mesmo que em comunidades pequenas de pesquisadores, esse tipologia de avaliação não funcione bem.

No Brasil há outro agravante, relacionado a pouca literatura sobre essa modalidade de avaliação. Numa busca simples por termos como "avaliação aberta de artigos" ou "avaliação aberta pelos pares" ou mesmo "open Peer Review" em sites brasileiros no Google Acadêmico no máximo encontram-se 114 documentos, que nem sempre tratam do assunto, mas apenas mencionam o termo, além de citações de artigos estrangeiros.

Nesse contexto, o presente estudo tem por finalidade estudar o processo de avaliação aberta pelos pares, de forma a trazer embasamento teórico voltado ao editor científico, que possibilite a decidir pela adesão ou não desta modalidade. Contribuir com a discussão sobre a CA, especificamente ao RAP, de forma a atender a pouca literatura sobre esse tema, que pode ser importante nas publicações em acesso aberto.

\section{PROCEDIMENTOS METODOLÓGICOS}

O estudo se enquadra como exploratório, na medida em que visa a proporcionar maior familiaridade a Revisão Aberta pelos Pares (RAP), de forma a explicitar os seus conceitos, alinhados a definição de Gil (2008). Da mesma forma em que se caracteriza como estritamente qualitativo, com técnica de coleta de dados baseado em levantamento bibliográfico e pesquisa documental, no qual artigos, livros e páginas web são as unidades de informação.

A principal fonte de coleta foi o Google Acadêmico, visto abranger uma vasta coleção de periódicos e livros, que de acordo com Harzing (2019) ainda se mantém como uma boa opção para levantamentos bibliográficos, tendo a vantagem de ser de acesso livre, comparando-se com Web Of Science, Scopus, CrossRef e outros. Assim, cobre-se uma vasta quantidade de fontes de informação, principalmente as de acesso aberto, como orientado pela Ciência Aberta.

A busca se deu prioritariamente no idioma inglês, visto que a literatura técnico-científica é predominantemente nesse idioma, principalmente nessa área. Com isso, o resultado de busca possibilitou recuperar os documentos completos para análise, buscando os conceitos e tendências relacionadas com a Ciência Aberta, na medida em que o tema pode ocorrer em outros contextos.

Entretanto, como a avaliação é uma atividade intermediária ao fluxo editorial, pode ser que não seja totalmente explorado na literatura técnico científica, apresentando certas restrições. Assim, 
o presente estudo se apresenta como inicial, não tendo a intenção de ser extenso e abrangente, mas contribuir com a discussão na área de editoração científica.

\section{RESULTADOS}

A questão da avaliação aberta não é algo novo voltado exclusivamente à Ciência Aberta (CA), tanto que, Van Rooyen et al (1999) revelam que autores e avaliadores conhecerem as identidades de ambos pode não influenciar na qualidade dos pareceres, mas que pode afetar a probabilidade dos avaliadores declinaram do convite para revisão. Esse ponto revela o receio, entre outros pontos, que ainda cercam a avaliação aberta, como mudança no processo de avaliação científica. Já Walsh et al (2000) verificaram que geralmente os avaliadores afirmam não se importarem com a avaliação aberta, mas aprovam mais, sendo mais corteses, com melhor qualidade de parecer.

Falagas (2007), discutindo a abertura das ciências, já advogava pela mudança no processo de avaliação pelos pares, de forma a torná-la mais transparente. Com isso, revela a preocupação das mudanças na ciência, principalmente com a ciência aberta e seu impacto na comunicação científica. Cabe destacar que a mudança da avaliação cega ou duplamente cega para a aberta, com a publicação dos pareceres, é mais política que técnica, na medida em que tecnologicamente não há empecilhos para a adesão. Bravo et al (2019) destaca que a Revisão Aberta pelos Pares (RAP) ainda é vista com desconfiança, apesar dos benefícios, pois muitos revisores sentem-se desprotegidos do véu da anonimidade, revelando as fortes questões culturais existentes no processo de publicação.

Schmidt (2018) apresenta questões para discussão acerca da RAP que merecem ser discutidos, sendo as principais:

- Não há uma única modalidade: questões ainda em aberto, como se o parecer será publicado se o trabalho for rejeitado ou se o parecerista e autores vão discutir o parecer, entre outros;

- Fomenta a confiança mútua, o respeito e a abertura às críticas: a avaliação torna-se um diálogo para melhoria do trabalho, em que os leitores podem acompanhar as recomendações e resultados;

- Proporciona qualidade construtiva e a transparência, com responsabilidade: a publicação dos pareceres garantem a transparência e apoia a melhoria do trabalho, numa contribuição construtiva.

- Possibilita o reconhecimento aos revisores e torna as avaliações citáveis: Pareceres publicados dão visibilidade aos avaliadores, possibilitando que esses relatórios possam ser citados.

Já Ross-Hellauer e Görögh (2019) apresentam uma lista de conselhos para a adoção da RAP, de forma a facilitar a sua implementação, em um apoio a política de RAP:

$\Rightarrow$ Definir as metas:

$\rightarrow$ Definir quais as metas a serem alcançadas pela RAP;

$\rightarrow$ Conhecer todas as possibilidades ofertadas pela RAP:

- Identificação dos autores e pareceristas; 
- Publicação da avaliação;

- Possibilidade da avaliação pela comunidade;

- Possibilidade de interação entre autor e avaliador;

- Disponibilização do pré-print ;

- Comentários pós-publicação;

- Revisão desacoplada, em que a avaliação é feita externamente a publicação.

- Implementar apenas o que for adequado a revista.

$\Rightarrow$ Ouvir a comunidade:

- Atender as diferenças entre comunidades;

- Considere consultar os leitores, autores e avaliadores;

- Envolver os stakeholders no processo.

\section{$\Rightarrow$ Planejar tecnologias e custos:}

- Avaliar as tecnologias que podem ajudar no processo;

- Avaliar os custos e benefícios;

- Considerar opções alternativas para testa

$\Rightarrow$ Ter uma abordagem pragmática:

- Definir prioridades;

- Testar com pilotos.

$\Rightarrow$ Ter plano para divulgação dos conceitos:

- Repassar ideia a comunidade;

- Dar atenção a comunicação e terminologia.

$\Rightarrow$ Avaliar desempenho:

- Ter claramente o que vai verificar como desempenho;

- Aceitar que mudanças levam tempo;

- Apresentar para a comunidade as avaliações.

$\Rightarrow$ Identidades abertas para autores e avaliadores:

- Ter estratégias para ter um corpo de avaliadores que aceitam essa modalidade;

- Estar alerta para interações conflituosas;

- Proporcionar identificação completa dos autores e avaliadores, como o ORCID para aumentar a visibilidade da avaliação;

- Testar primeiro ou colocar a identidade aberta opcional.

$\Rightarrow$ Publicar a avaliação junto a artigo aceito:

- Encontrar melhor forma para publicação da avaliação, como documento adicional ou na apresentação do artigo;

- Estar ciente dos desafios na publicação dos pareceres.

$\Rightarrow$ Participação aberta, desde o pré-print ao pós-print:

- Decidir quem pode comentar;

- Fomentar a participação da comunidade.

$\Rightarrow$ Interação aberta:

- Definir o fluxo de interação a ser implementado; 
- Estar ciente da carga adicional de trabalho.

Barret (2019), por sua vez, revela que a RAP pode apoiar o aprendizado para novos pesquisadores, na medida em que ao publicar as avaliações, pareceristas em potencial podem aprender como fazê-lo. Esse ponto alinha ao pensamento que a RAP é uma ferramenta de aprendizado para outros avaliadores, visto que indícios revelam que pareceres abertos são melhor apresentados, com maior cortesia e colaboração para melhoria do trabalho.

Um dos pontos relacionados ao processo de avaliação é que o parecerista deve ser conhecedor do assunto do trabalho a ser avaliado. Assim, com o RAP, um canal de diálogo pode ser efetuado entre parecerista e autor, de forma a melhorar o trabalho. Assim, Lobo (2019) sugere que os avaliadores abertos podem ser convidados a ser co-autores, mesmo que esse procedimento requeiram código de ética bem estruturado, na medida em que colaboram como texto, se aberto a discussão entre autores e avaliadores.

Cabe destacar que a avaliação aberta é complexa e possui várias questões que ainda estão em discussão ou que não são totalmente conceituados. Da mesma forma que possui vários pontos para customização, com uma flexibilidade que possibilita ao editor adequar a avaliação aberta da forma que atende melhor a revista.

\section{CONCLUSÃO}

As discussões sobre a Revisão Aberta pelos Pares estão se iniciando no Brasil. Apesar de teoricamente promissora apresenta complexidades a serem contornadas, em especial os conflitos de interesse. Amaral e Príncipe (2018) apresentam alguns desafios e reflexões que acometem a implantação imediata desta modalidade no país, destacando os problemas das relações acadêmicas que poderão advir entre autores e avaliadores.

A mudança do paradigma tradicional, ou seja, da modalidade de avaliação fechada para aberta envolve mudanças em processos estabelecidos e aceitos pela comunidade envolvida na publicação científica. Com isso, pode trazer desconfianças que pode acometer a adesão, requerendo ações proativas dos Editores, de forma a produzir um ambiente favorável a implementação do RAP.

É possível que esta "abertura" seja irreversível haja vista que cerca de 70\% (ROMERO, 2017) da ciência publicada no mundo não tem reprodutibilidade. A abertura dos dados científicos e dos avaliadores daria transparência e seria uma maneira de amenizar o enorme dispêndio de investimentos públicos na pesquisa científica sem retorno à comunidade.

Por fim, estudos mais aprofundados sobre o RAP no Brasil serão necessários, visto que a atual forma de avaliação fechada trás inúmeras críticas, mesmo sendo a mais aceita atualmente. Estudos sobre a avaliação aberta acompanha as tendências de abertura das ciências, com procedimentos mais transparentes e democráticos, fornecendo subsídios para editores avaliarem a sua adoção. 


\section{REFERÊNCIAS}

ALBAGLI, Sarita; CLINIO, Anne; RAYCHTOCK, Sabryna. Ciência Aberta: correntes interpretativas e tipos de ação. Liinc em Revista, v. 10, n. 2, 2014. Disponível em: <https://doi.org/10.18617/liinc.v10i2.749>. Acesso em: 02 set. 2019.

AMARAL, Janaynne Carvalho do; PRÍNCIPE, Eloísa. Ciência aberta e revisão por pares: aspectos e desafios para a participação da comunidade em geral. Cadernos BAD, n. 1, p. 320-325, 2018. Disponível em: <https://www.bad.pt/publicacoes/index.php/cadernos/article/view/1934>. Acesso em: 02 set. 2019.

BANKS, George C. et al. Answers to 18 questions about open science practices. Journal of Business and Psychology, v. 34, n. 3, p. 257-270, 2019. Disponível em: <https://doi.org/10.1007/s10869-018-9547-8> . Acesso em: 02 set. 2019.

BARRETT, Spencer CH. Proceedings B 2018: the year in review. Proceedings of the Royal Society B: Biological Sciences,v. 286, n. 1894, 2 Jan. 2019. Disponível em: <https://doi.org/10.1098/rspb.2018.2590>. Acesso em: 02 set. 2019.

BRAVO, Giangiacomo et al. The effect of publishing peer review reports on referee behavior in five scholarly journals. Nature communications, v. 10, n. 1, p. 322, 2019. Disponível em: <https://www.nature.com/articles/s41467-018-08250-2>. Acesso em: 02 set. 2019.

COLE, Jonathan R. Balancing acts: Dilemmas of choice facing research universities. Daedalus, v. 122, n. 4, p. 1-36, 1993. Disponível em: <https://www.jstor.org/stable/20027197?seq=1\#page_scan_tab_contents>. Acesso em: 02 set. 2019.

DAVID, Paul A. Reputation and Agency in the Historical Emergence of the Institutions of 'Open Science'. In: NATIONAL ACADEMY OF SCIENCES COLLOQUIUM ON THE ECONOMICS OF SCIENCE AND TECHNOLOGY, 1995, Irvine, Ca. Proceedings... . Irvine: Beckman Center, 1995. Disponível em: <https://www.researchgate.net/publication/239063416_Reputation_and_agency_in_the_historical_emergence_of_the_institutions_of_Open_Science>. Acesso em: 02 set. 2019.

FALAGAS, Matthew E. Peer review in open access scientific journals. Open medicine, v. 1, n. 1 , p. e49 - e51, 2007. Disponível em: <https://www.ncbi.nlm.nih.gov/pmc/articles/PMC2801911/>. Acesso em: 02 set. 2019.

GIL, Antonio Carlos. Métodos e técnicas de pesquisa social. 6. ed. São Paulo : Atlas, 2008. 200 p. ISBN 978-85-224-5142-5.

HARZING, Anne-Wil. Two new kids on the block: How do Crossref and Dimensions compare with Google Scholar, Microsoft Academic, Scopus and the Web of Science?. Scientometrics, p. 1-9, 2019. Disponível em: <https://link.springer.com/article/10.1007/s11192-019-03114-y>. Acesso em: 02 set. 2019.

LOBO, Matheus P. 2019. Peer Reviewers as Co-authors. OSF Preprints. 27 maio 2019. Disponível em: <https://osf.io/jtnq2/>. Acesso em: 02 set. 2019. DOI:10.31219/osf.io/jtnq2. 
MILHAM, Michael P.; KLEIN, Arno. Be the change you seek in science. BMC biology, v. 17, n. 1, p. 27, 2019. Disponível em: <https://bmcbiol.biomedcentral.com/articles/10.1186/s12915-019-0647-3>. Acesso em: 02 set. 2019.

PARTHA, Dasgupta; DAVID, Paul A. Toward a new economics of science. Research policy, v. 23, n. 5, p. 487-521, 1994. Disponível em: <https://www.sciencedirect.com/science/article/pii/0048733394010021>. Acesso em: 02 set. 2019.

ROMERO, Flávio. A Ciência na idade das trevas. Estadão, São Paulo, 27 jun. 2017. Disponível em: <https://cultura.estadao.com.br/blogs/estado-da-arte/a-ciencia-na-idade-das-trevas/> Acesso em: 02 set. 2019.

ROSS-HELLAUER, Tony; GÖRÖGH, Edit. Guidelines for open peer review implementation. Research Integrity and Peer Review, v. 4, n. 1, p. 4, 2019. Disponível em: <https://researchintegrityjournal.biomedcentral.com/articles/10.1186/s41073-019-0063-9> Acesso em: 02 set. 2019.

SCHMIDT, Birgit et al. Ten considerations for open peer review. F1000Research, v. 7, 2018. Disponível em: <https://f1000research.com/articles/7-969> Acesso em: 02 set. 2019.

SILVA, Jaime A. Teixeira da. Challenges to open peer review. Online Information Review, v. 43, n. 2, p. 197-200, 2019. Disponível em: <https://www.emerald.com/insight/content/doi/10.1108/OIR-04-2018-0139/full/html> Acesso em: 02 set. 2019.

VAN ROOYEN, Susan et al. Effect of open peer review on quality of reviews and on reviewers' recommendations: a randomised trial. BMJ, v. 318, n. 7175, p. 23-27, 1999. Disponível em: < https://doi.org/10.1136/bmj.318.7175.23> Acesso em: 02 set. 2019.

WALSH, Elizabeth et al. Open peer review: a randomised controlled trial. The British Journal of Psychiatry, v. 176, n. 1, p. 47-51, 2000. Disponível em: < https://www.cambridge.org/core/journals/the-british-journal-of-psychiatry/article/open-peer-review-a-randomised-controlled-trial/1F81447FC67B3BAFDCCCC E82B6C7A187> Acesso em: 02 set. 2019. 